\title{
Sports videogames, \\ un ambiente virtual para la enseñanza de las normas deportivas
}

\author{
Sports videogames, a virtual environment for teaching sports rules
}

\author{
Sonia Rocio Corredor Castro"
}

\section{Resumen}

\begin{abstract}
Este artículo describe una experiencia de implementación de videojuegos deportivos en dos colegios oficiales de Cundinamarca. El propósito consistió en demostrar que el videojuego al ser utilizado como herramienta didáctica facilita la comprensión de las normas deportivas, para ello se realizaron interacciones con aplicaciones de pre-test y post-test con pautas de observación para hacer una validación de los aspectos relacionados con la pedagogía y la práctica deportivas. Los resultados apuntan a probar que la herramienta tiene efectos educativos importantes y que sirve como apoyo al profesor en el desarrollo de la clase de educación fisica. Participaron estudiantes de educación básica secundaria en los ámbitos Distrital y municipal.
\end{abstract}

Palabras Clave: Educación fisica, normas deportivas, pedagogia deportiva, videojuego.

\section{Abstract}

This article describes a sports videogames implementation experience in two Cundinamarca's public schools. This intention consisted of showing that if the sports videogames are used as a didactic tool then they facilitate the understanding of the sports rules. To do this, several interactions with pre-test and post-test applications and observation guidelines werw carried out for validating aspects related with the sports pedagogy and sports practice. The results obtained may demonstrate that this tool has very important educational effects and supports the teacher in the physical education class development. In this class participated the secondary education pupils.

Key words: $\quad$ Physical education, sports rules, sports pedagogy, videogames.

Fecha de recepción:20 de diciembre de 2004.

Fecha de acepatación:29 de julio de 2005.

- Investigación producto de la Práctica Pedagógica y Didáctica III de la Facultad de Educación Física de la Universidad Pedagógica Nacional, 2004. Estudiantes coinvestigadores: Carolina Caballero, Claudia Espinosa, Cristian Gamboa, Francisco Henao, . Jonathan Leal, Stella Osorio, Ingrid Peña, John Jairo Sandoval.

*. Profesora de la Facultad de Educación Física. Asesora del proyecto de investigación. 


\section{Antecedentes}

El uso de la tecnología en la vida profesional y cotidiana supone una alteración en los parámetros temporales y espaciales y en sus propios contenidos. Por este motivo los docentes se ven sometidos a modificaciones muy importantes en las formas de acceso al conocimiento, a las relaciones, a la informática y a la distribución de su tiempo y su espacio. Los profesores ya no se ven como los que "saben", sino que son las nuevas generaciones las que dominan la tecnología que poco o nada son utilizadas por los docentes. Los jóvenes acceden de forma fácil y sencilla al manejo de los nuevos ambientes virtuales como fuente de información y conocimiento. En el contexto educativo se presentan estos problemas, los cuales han venido abordándose mediante cambios en las formas de aprendizaje y en los métodos de enseñanza.

En la actualidad se ha observado, y en algunas ocasiones con preocupación, la invasión de videojuegos y nuevas tecnologías que saturan a la niñez y la adolescencia con diferentes clases de información sin cualificar su contenido.

Los niños y jóvenes de hoy se enfrentan a una revolución tecnológica que está transformando buena parte de los fenómenos económicos, políticos, sociales, cultura: les y educativos que forman parte de su vida diaria. Los cambios derivados de esta revolución tienen un impacto importante en la manera en que ellos juegan y aprenden, e incluso en cómo viven y cuáles son sus expectativas de trabajo en el futuro. Para este nuevo milenio, las habilidades básicas como leer, escribir, realizar operaciones lógico-matemáticas para desarrollar un pensamiento creativo y crítico, no serán suficientes, pues en el interés de adaptarse a la nueva sociedad de la información, los estudiantes tendrán que aprender aptitudes especiales que les permitan trabajar, divertirse y convivir con estas nuevas tecnologías, si quieren sobrevivir en él.

Por ello se considera que para los educadores y en particular los docentes en educación física ha llegado el momento de incorporar y utilizar estas nuevas tecnologías en su quehacer pedagógico, como parte de la estrategia metodológica que les permita ampliar, transferir, mejorar, crear o transformar los conocimientos que la disciplina deportiva debe impartir para mejorar su aprendizaje.

\section{Surgimiento del proyecto}

Se escoge el videojuego como herramienta tecnológica por su gran aceptación y motivación en los niños y adolescentes; además, en el contexto académico encontra- mos la necesidad de integrar saberes y prácticas entre las áreas curriculares, por cuanto en la mayoría de las instituciones se cuenta con un aula de informática y un área deportiva. Para establecer una correlación entre las prácticas del deporte formativo se seleccionan: el baloncesto, como deporte de conjunto, presente en los programas de educación física, y por el interés de algunos de los coinvestigadores; y el tenis de campo, como deporte individual de poca o ninguna práctica en los colegios.

\section{Diseño del proyecto}

A partir del proyecto planteado y de los esbozos iniciales, se inicia con un proceso de reflexión y cuestionamiento sobre la aplicabilidad y viabilidad de los diferentes videojuegos que posibilitarán el aprendizaje y la comprensión de las normas deportivas; continúa con la interacción de los coinvestigadores en diferentes videojuegos para vivenciar y visualizar qué tan claras son las normas deportivas allí expuestas -se interactuó con videojuegos de diferentes clases, como simuladores de estrategia, de roles y de retos-; finalmente, se determina trabajar con un deporte grupal y otro individual, y de los manipulados se escogieron baloncesto y tenis de campo, ya que además de ser llamativos visualmente, también están relacionados con las clases de educación física en los colegios de secundaria.

Luego de interactuar con los videojuegos surgen interrogantes que llevan a buscar fuentes bibliográficas que se relacionen con videojuegos, aprendizaje significativo, comprensión, feedback extrínseco ${ }^{2}$ y que van ampliando la visión sobre los conceptos que trae implícito este nuevo método que se quiere implementar.

\section{Contenidos del proyecto}

\section{Problema}

$\mathrm{Al}$ abordar el tema del reglamento deportivo de un deporte determinado en las clases de educación física, surge la inconformidad, el tedio y el aburrimiento de los estudiantes debido a que ellos sólo quieren la práctica directamente; centrar su atención en las exposiciones teóricas se constituye en un reto para el maestro. Así mismo, en el contexto social ellos encuentran muchas ofertas de juegos interactivos relacionados algunos con las prácticas deportivas, lo cual incrementa los niveles de desmotivación y gusto por aprender la teoría de los deportes. Entonces, cabe preguntarse, ¿existen diferencias significativas en la comprensión del reglamento de 
un deporte, en un grupo de estudiantes que interactúa con un videojuego, y la técnica de feedback extrínseco, frente a la manera en que afrontan las situaciones de juego?

\section{Objetivos}

\section{General}

Identificar en los videojuegos y en la técnica del feedback extrínseco una herramienta pedagógica que fortalece y facilita la comprensión de los reglamentos de baloncesto $y$ tenis de campo en los estudiantes de grado sexto del Instituto Pedagógico Nacional (IPN) y del Colegio Nacional Emilio Cifuentes (CNEC).

\section{Específicos}

1. Rescatar el gusto e interés de los estudiantes para interactuar con videojuegos deportivos.

2. Establecer a través de comparativos las faltas, el terreno de juego, el juzgamiento, y las violaciones a los reglamentos que son recurrentes en la práctica y uso de los video-juegos.

3. Implementar pruebas de entrada que permitan identificar los conocimientos previos manejados por los estudiantes con respecto a los reglamentos de baloncesto y tenis de campo.

4. Estructurar la técnica de "observación" para registrar el desempeño de los estudiantes en situaciones de juego real.

5. Incrementar el gusto e interés por el aprendizaje del reglamento de baloncesto y de tenis de campo, mediante el uso de ambientes virtuales con feedback extrínseco en la simulación del baloncesto y el tenis de campo.

6. Analizar la propuesta investigativa a través de la aplicación de una prueba de salida que permita valorar la adquisición de aprendizajes.

7. Sistematizar los resultados de la experiencia pedagógica de investigación.

\section{Metodología}

\section{Modelo de Investigación}

El modelo a seguir en esta investigación es el cuasiexperimental, por cuanto los estudiantes no son asignados al azar a los cursos, sino que dichos cursos ya estaban conformados antes del experimento. Organizados por el horario de clases de informática y de educación física, por el grado escolar y por el orden de lista. Se definen grupos de igual número de participantes, unos reciben feedback extrínseco -entendido como la entrega de información externa al estudiante acerca de su desempeño con el propósito de mejorarlo en el futuro-; los otros no. La comparación, la interpretación y el análisis se efectúan a todos los grupos.

\section{Población}

Niños y niñas en edades de 11 a 13 años de sexto grado de educación básica secundaria. Del Instituto Pedagógico Nacional se elige un curso con 32 estudiantes y al grupo que está en informática y recibe el tratamiento de feedback extrínseco en baloncesto. Del Colegio Nacional Emilio Cifuentes se seleccionan dos cursos: uno de 40 estudiantes para baloncesto y otro de 42 estudiantes para tenis de campo. Cada curso se divide en dos grupos, A y B; los grupos A reciben el tratamiento experimental, los grupos B, no. Total: 114 estudiantes.

\section{Instrumentos de recolección de datos}

\section{Encuesta diagnóstica}

Preguntas para determinar el grado de interés, aceptación o uso de los videojuegos aplicada tanto a estudiantes como a docentes de las instituciones mencionadas, variando la formulación de algunos ítems de acuerdo con la familiaridad de los estudiantes con respecto a los videojuegos.

\section{Observación}

Registro sistemático, válido y confiable de actitudes, reacciones y comportamientos en diferentes situaciones de juego real que tienen los estudiantes dentro del campo de juego de baloncesto o de tenis de campo. Elaborado en forma de diario de campo antes y después de haber realizado la interactividad de cada videojuego.

\section{Prueba piloto}

Preguntas con alternativas de respuesta, señalización, relación y falso o verdadero acerca de lo que conocen, han observado o han practicado los estudiantes sobre los reglamentos de baloncesto ( 15 ítems) y tenis de campo (11 ítems). La prueba o test piloto es aplicada antes y después de la interactividad con el videojuego; la comparación entre el pretest y el postest permite establecer el grado de los objetivos que se lograron. 


\section{Fases del proyecto}

Para dar respuesta a los objetivos propuestos de la investigación se plantean tres fases:

\section{Primera Fase}

a. Delimitación institucional: es decir, el sitio donde se realiza la investigación; se prefiere el Instituto Pedagógico Nacional, ubicado en la Calle 127 con Cra. 9, por ser adscrito a la Universidad Pedagógica Nacional.

Además para hacer un cuadro comparativo a nivel cognitivo entre un colegio de Bogotá y otro de un muni cipio aledaño, se selecciona el Colegio Nacional Emilio Cifuentes de Facatativa, por ser la institución donde trabaja la asesora del proyecto.

b. Delimitación de la población: es la población objeto de la investigación descrita antes.

c. Selección de los deportes: se cuenta con el dominio deportivo por parte de los coinvestigadores y con el programa de educación física de las instituciones seleccionadas.

d. Marco legal y teórico: información sobre juego, deporte, aprendizaje significativo, feedback, comprensión, concepto, evolución y relación entre educación $\mathrm{y}$ videojuegos.

e. Diseño y aplicación de encuestas a docentes: sé aplican no sólo a los docentes mencionados, sino también a los de otros colegios, otras áreas y otros niveles.

\section{Segunda fase}

a. Instalación del videojuego de baloncesto: en los dos colegios existe un aula especializada para la clase de informática con computadores que tienen unidad de $\mathrm{CD}$, fundamental para esta investigación; además, se cuenta con el apoyo por parte de las personas encargadas.

b. Diseño y aplicación de encuesta a estudiantes: contiene 10 preguntas de selección, de completar, de relación entre juego real y juego virtual de baloncesto.

c. Diseño y aplicación de la prueba piloto No. 1 (pretest) de baloncesto: test de entrada en el que se busca qué tanto saben o conocen los estudiantes del deporte de baloncesto; aplicada antes de la interactividad con el videojuego.

d. Diario de campo No. 1: observación de un partido en el campo de juego de baloncesto antes de realizar la interacción con el videojuego en cada uno de los colegios. e. Interacción con el videojuego de baloncesto: los estudiantes se ubican por parejas en el computador y los coinvestigadores aplican el tratamiento del feedback extrínseco al grupo seleccionado de cada colegio, en las tres sesiones que se desarrollaron.

f. Aplicación de la prueba piloto No. 2 (postest): una vez terminadas las sesiones de interactividad se aplica el test de salida.

g. Diario de campo No. 2: observación de un partido en el campo de juego de baloncesto después de realizar la interacción con el videojuego en cada colegio.

h. Adelantos en antecedentes, marco legal, actividad física, aspecto pedagógico y ambiente virtual.

i. Sistematización y análisis de los instrumentos de recolección de datos: se tabulan las respuestas señaladas por los niños tanto de las encuestas como de las pruebas pilotos No. 1 y No. 2, enmarcadas en las categorías de análisis de reglamento: terreno de juego, recurso humano, faltas y violaciones; se esquematizan los diarios de campo.

\section{Tercera fase}

a. Instalación del videojuego de tenis de campo: por motivo de remodelación de las canchas deportivas del IPN solo se aplica esta fase al Colegio Nacional Emilio Cifuentes.

b. Diseño y aplicación de encuesta a estudiantes: contiene 10 preguntas de selección, de completar, de relación entre juego real y juego virtual de tenis de campo.

c. Diseño y aplicación de la prueba piloto No. 3 (pretest) de tenis de campo: test de entrada, en el cual se busca saber qué tanto saben o conocen los estudiantes del deporte de tenis de campo, aplicada antes de la interactividad con el videojuego.

d. Diario de campo No. 3: observación de un partido en la cancha acondicionada para tenis de campo antes de realizar la interacción con el videojuego.

e. Interacción con el videojuego de tenis de campo: los estudiantes se ubican por parejas en el computador, y los coinvestigadores aplican el tratamiento del feedback extrínseco al grupo seleccionado, en las tres sesiones que se desarrollaron.

f. Aplicación de la prueba piloto No. 4 (postest): una vez terminadas las sesiones de interactividad se aplica el test de salida.

g. Diario de campo No. 4: observación de un partido en la cancha acondicionada para tenis de campo después de realizar la interacción con el videojuego. 
h. Sistematización y análisis de los instrumentos de recolección de datos. Se tabulan las respuestas señaladas por los niños tanto de las encuestas como de las pruebas pilotos No. 3 y No. 4 , enmarcadas en las categorías de análisis de reglamento a saber: terreno de juego, juzgamiento, generalidades, puntuación y servicio; se esquematizan los diarios de campo.

\section{Resultados}

Las gráficas 1,2 y 3 presentan el comportamiento porcentual de las respuestas obtenidas de los estudiantes del IPN y del CNEC antes y después de interactuar tanto con el videojuego de baloncesto como con el de tenis de campo y el tratamiento de feedback extrínseco. Se evidencia un importante incremento en los porcentajes de las respuestas correctas.

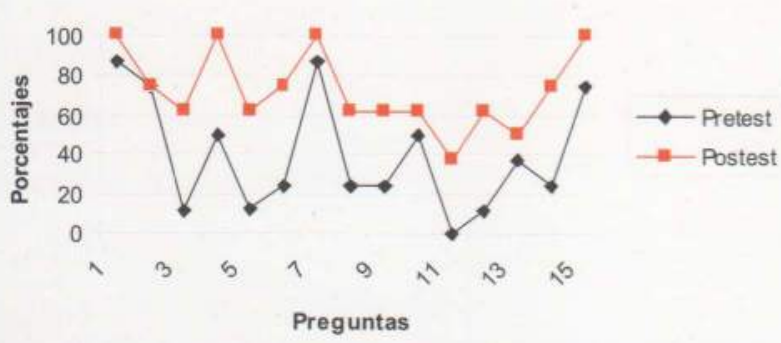

Gráfico 1. Correlación de respuestas pretest y postest baloncesto IPN.

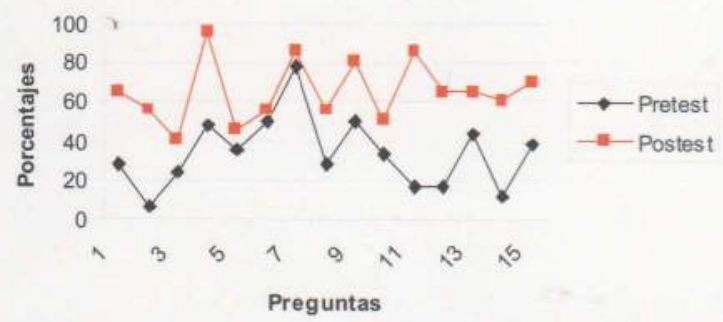

Grảfico 2. Correlación de respuestas pretest y postest baloncesto CNEC.

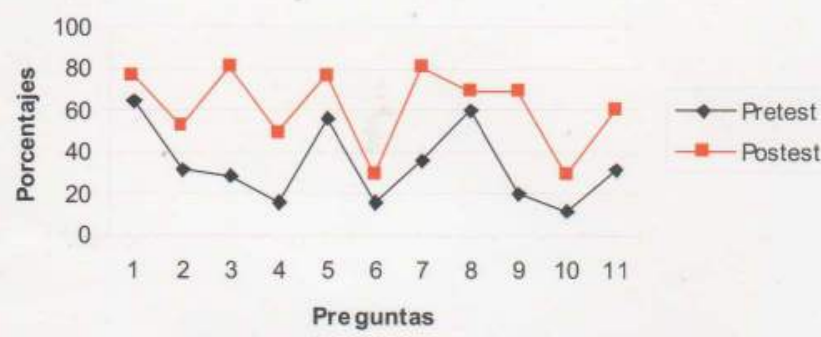

Gráfico 3. Correlación de respuestas pretest y postest tenis de campo.
La gráfica 4 presenta el comportamiento porcentual del consolidado de respuestas obtenidas por colegio en las pruebas antes y después de interactuar con el videojuego de tenis de campo y la aplicación de feedback extrínseco. Se verifica el incremento porcentual en las respuestas correctas.

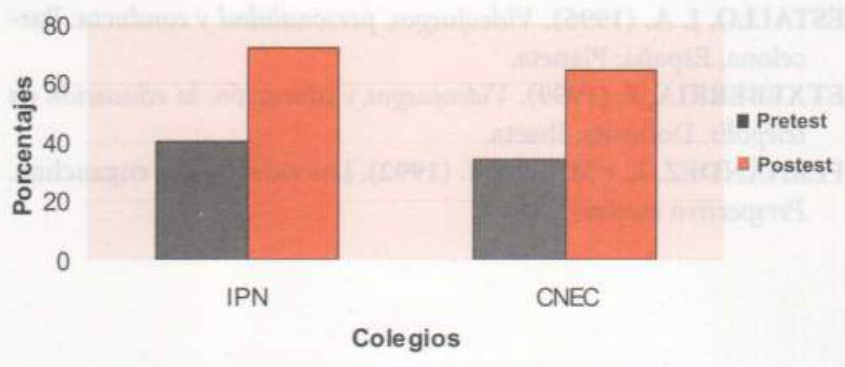

Gráfico 4. Resultado general comparativo pretest y postest.

\section{Aportes}

El grupo de práctica pedagógica que lidera el proyecto Sports videogames un ambiente virtual para la enseñanza de las normas deportivas rescata los beneficios que tienen los videojuegos deportivos, considerados después de esta investigación como poderosa herramienta pedagógica que motiva y promueve el aprendizaje de reglamentos deportivos, para la clase de educación física. Así mismo, el proyecto demuestra que los docentes necesitan hacer uso de estrategias y metodologías que respondan a las necesidades que actualmente demandan los estudiantes. También presenta el feedback extrínseco como herramienta metodológica, que permite enriquecer los preconceptos de los estudiantes, con el fin de fortalecer el proceso de enseñanza-aprendizaje en cualquier área curricular. Finalmente, los educadores físicos no deben desconocer las nuevas tecnologías para el apoyo, proyección y cualificación de su quehacer 


\section{Bibliografía}

ALVES DE MATTOS, L. (1963). Compendio de la Didáctica General. Buenos Aire. Kapelusz.

CALVO S, A. M. (1998). Que se sabe de los videojuegos: juguetes y juegos. Valencia, España. Dossier,

ESTALLO, J. A. (1995). Los videojuegos: juicios y prejuicios. Barcelona, España. Planeta,

ESTALLO, J. A. (1996). Videojuegos, personalidad y conducta. Barcelona, España: Planeta.

ETXEBERRIA, F. (1999). Videojuegos y educación: la educación en telépolis. Donosita. Ibaeta.

FERNÁNDEZ, L. y MARÍN, I. (1992). Los videojuegos enganchan. Perspectiva escolar.

\section{Notas}

1 Feedback extrínseco o aumentado es aquel que proviene de una fuente externa, es decir, que se presenta cuando el profesor estimula, corrige o proporciona información al estudiante.
FIELINI, F. Play as acquisition of mental structures: the case of videogames: Study of sociology, Calabria. Italia.

GROSS, B. (1998). Jugando con videojuegos: educación y entretenimiento. Bilbao. Desclee de Brouwer,

KRIDSON, D., y MORRISON, C. (1997). Qualitative analysis of human movement. Champaign III. Human Kinetics.

PEÑA, Bernardo. (1986). Educación e informática. Bogotá. Colciencias.

PÉREZ, M., $y$ LÓPEZ, J. Los videojuegos como nueva realidad social y cultural. En: Infancia y sociedad. No. 1/20

STONE WISKE, M. (1999). La enseñanza para la comprensión. México. Paidós. 